\title{
CORRECTIONS TO BUNGE'S \\ FOUNDATIONS OF PHYSICS (1967)
}

\begin{abstract}
Bunge's new book ${ }^{1}$, taken as a whole, is an excellent piece of work - the sole exception being the chapter on quantum mechanics (QM). To say that the other chapters are excellent is not to say that they are on the highest level now attainable but that they are an excellent approximation to that level. This is more than can be said of most textbooks in physics, and certainly more than can be said of any other book on foundations of physics. It follows that the book has a good chance to become a standard work of reference to all interested in the subject. It is precisely for this reason that the errors and shortcomings in the book should be pointed out by fellow workers and particularly by those who have a longer and/or more detailed experience in this difficult field of research.
\end{abstract}

\section{MATHEMATICAL ERRORS}

1. (Galilei, Lorentz, Einstein group) "The Galilei group was eventually embedded in the Lorentz group, in turn included in the group(oid) of arbitrary coordinate transformations" (p. 87).

The Galilei group is not a subgroup of the Lorentz group and hence cannot be "embedded" in it; it has been replaced by the latter. From the mathematical point of view, the proper Galilei group is a reducible representation (in the group theoretical sense) of an abstract algebraic group (physical interpretation: constant velocity group), while the proper Lorentz group is an irreducible representation of that algebraic group. ${ }^{2}$

Similarly, the Lorentz group is not a subgroup of ("included in") the Einstein group of GRT since there are no global frames of reference in the latter and a fortiori no such frames that could be connected by Lorentz transformations. ${ }^{3}$

2. (Spacetimes) "Df.1. Spacetime: $E^{3+1}={ }_{\mathrm{dr}} E^{3} \times T^{\prime}$ (p. 184).

This is not a definition but a contradiction: Minkowski space $\left(E^{3+1}\right)$ is 
not the direct product of $E^{3}$ (Euclidean space) and $T$ (time). In fact, $E^{3} \times T$ is the spacetime of Newtonian physics.

3. (Coordinate transformations) "Df.2. If $x^{\mu}$ and $x^{\prime \mu}$ are local coordinates in the neighborhoods of some points $x, x^{\prime} \in M^{4}$ and these neighborhoods have a non-empty intersection, and if $f^{\mu}$ are $1: 1$ functions of the class $C^{r}$, then $x^{\prime \mu}=f^{\mu}\left(x^{\nu}\right)$ is called a coordinate transformation of class $C^{\boldsymbol{r} \prime \prime}$ (p. 219).

This looks like a quotation from high standard text book but it is wrong all the same. The definiens does not define a coordinate transformation, i.e. a transition from one mapping to another one, but a mapping of $M^{4}$ onto itself: in a coordinate transformation $\left\{x^{\mu}\right\}$ and $\left\{x^{\prime \mu}\right\}$ would refer to the same point of $M^{4}$.

\section{ERRORS IN LOGIC}

4. (Transformation properties) "In fact, establishing whether or not a given theory has certain transformation properties is a purely conceptual operation" (p. 54).

Holy Simplicity! In fact, this statement is not true, unless the transformation properties of all terms in the fundamental equations follow from the definition of these terms. This condition is not satisfied by either Newton's or Maxwell's equations, the Newtonian force F and the Maxwell field vectors being primitives! That they transform as 3-vectors under spatial rotation is indeed implied, but their kinematic transformation properties are not to be read off from the fundamental equations but have to be postulated. In particular, as far as mathematics and logic are concerned, we can always postulate that there is no kinematic covariance group (single preferential frame theories). It follows that Galilei covariance of Newton's equations and Lorentz covariance of Maxwell's equations are physical postulates. That these postulates are not formulated in the object language does not render them metatheoretical: all syntactic and semantic axioms refer to expressions of the object language and hence have to be stated in the (first) metalanguage. Metatheoretical statements must refer to these axioms and hence have to be formulated in the second metalanguage (meta-metalanguage).

The physical content of the postulate of Galilei covariance of Newton's equations can be seen from the fact that this postulate restricts the class 
of admissible laws of force: the mathematical expression for a force law must be invariant under the proper Galilei group! - Similarly, the physical content of the postulate of Lorentz covariance of Maxwell's equations can be seen from the fact that it leads to different predictions from those of the two alternatives (i.e., Galilei covariance and no kinematic group covariance). 4

5. (SR/CEM relation) "SR (Special Relativity) presupposes CEM (Classical Electromagnetism = Maxwell's Theory)" (p. 62).

In fact, it does nothing of the sort. If electromagnetic fields, and hence CEM, were non-existent, $c$-mechanics would still exist and be needed, $c$ being the limiting signal velocity. That SR did arise in connection with questions relating to CEM is nothing but a historical coincidence. Moreover, you cannot even deduce the Lorentz group from the Maxwell equations since the kinematic covariance group of the latter is not given but has to be postulated: there may be none (single preferential frame theory), see 4 above.

It follows that the axiomatic formulation of SR as given in the book, though not wrong, is utterly misleading in questions concerning the logic of intertheory relations.

6. (Missing axiom) "Df.9. A frame $k \in K$ such that the axioms $C E M 1$ to 2 above hold relative to it is called an inertial frame" (p. 164).

This is a definition allright, but the axioms referred to do not imply the existence of such a frame. Thus, an existence postulate is missing.

7. (Logical miracle) "We accept the stochastic interpretation of $\Psi$ and its components but do not postulate it: it shall be deduced right away" (p. 252).

As a matter of logic, you cannot deduce a probabilistic ("stochastic") statement except from a set of other such statements. If you show that a certain mathematical expression satisfies certain formal axioms (here: those of Kolmogoroff's theory) you have found a mathematical model of that theory. No extramathematical meaning is implied or deduced thereby.

It follows that the theorem Thm. 1 (p. 252), introducing the outdated prob $_{1}$ interpretation of $\mathrm{QM}$, is not a theorem but a semantic postulate, and a wrong one at that. 


\section{MISTAKES IN PHYSICS}

8. (p. 22) - 'kg' denotes a unit of mass, not of weight (a kind of force).

9. (Physical constants) "Boltzmann's constant $k$ and PlanCK's constant $h$ are numbers..." (p. 34).

Wrong: they are physical constants of non-zero physical dimensions, and hence they cannot be equated to numbers. - Cf. also 10 below.

10. (Are scales arbitrary?) "There are as many S's (scale-cum-unit systems) as we please" (p. 31).

Wrong again. Units are arbitrary (conventional), scales are rarely so. You cannot change the scale metric (definition of equality of intervals) of time without destroying the whole edifice of physics which employs the canonical time scale ${ }^{5}$ throughout. Indeed, if the time scale metric, and hence the velocity scale metric, were arbitrary you could use the scale transformation

$$
v^{\prime}=c t h(v / c)[t h=\text { tangens hyperbolicus }]
$$

to send the Galilei addition law

$$
v_{12}+v_{23}=v_{13}
$$

over into the Einstein addition law

$$
v_{12}^{\prime}+v_{23}^{\prime}=\frac{v_{12}^{\prime}+v_{23}^{\prime}}{1+v_{12}^{\prime} v_{23}^{\prime} / c^{2}}=v_{13}^{\prime}
$$

thus conjuring up (in Newtonian physics!) a velocity $c$ satisfying

$$
v+c=c .
$$

Moral: Beware of conventionalism, whether of the Poincare or the Carnap brand.

11. (Michelson experiment) "Thm.7 [i.e. $L=L_{0}\left(1-u^{2} / c^{2}\right)^{1 / 2}$ ] is sufficient to explain the null result of the Michelson experiment" (p. 193).

This is a concession to a popular fairy-tale. The truth is that the (original) Michelson experiment involved only one frame of reference and hence could not possibly test any kinematical theory. What it did test and 


\section{CORRECTIONS TO BUNGE'S 'FOUNDATIONS OF PHYSICS'}

verify is the invariance of the sum of to- and fro-time of light under spatial rotation, which is even less than isotropy of light propagation. ${ }^{6}$

12. (Mass in Newtonian physics) "In summary (a) the lack of difference between the inertial and the gravitational mass does not constitute a law of nature: the statement is just the negate of a tentative conjecture..." (p. 210).

By the same token we would have no laws of nature at all since every law of nature is the negate of some possible conjecture: if it were not it would be analytically true or false. You can avoid stating the law in explicit form, following Newton, but I do not think this is good form in axiomatics.

13. (Mass in $S R$ ) "there can be no relative [mass] $m$ unless there is a proper mass $m_{0}$ to begin with" (p. 201). "When a body is heated..., its increase $\Delta E$ in energy is accompanied by an increase in mass $\Delta m=\Delta E / c^{2}$. But if the radiation in a hollow cavity increases in energy by $\Delta E$, the field in it gains no mass although the walls do lose mass by the amount $\Delta m=\Delta E / c^{2 " \prime}$ (p. 202).

The first statement and the second part of the second statement are wrong. Put a hollow cavity with reflecting walls and an inbuilt electric radiator on a sensitive balance. The energy supplied to the radiator becomes radiation energy (standing electromagnetic waves or photons). SR predicts that the weight of the cavity increases by $g \Delta m=g \Delta E / c^{2}$, $\triangle E$ being the energy supplied, although neither the walls nor the radiator lose any mass since their respective temperatures remain constant. It also predicts that the inertial mass of the cavity has increased by $\Delta m$.

The book's mistake in this matter results from the mistaken belief that SR-mechanics does not apply to electromagnetic radiation. In point of fact, SR-mechanics includes the dynamics of zero-rest-mass particles: from the general equation

$$
E^{2}-c^{2} P^{2}=m^{2} c^{4}(=\text { invariant })
$$

you get

$$
E=c P \text { for } m=0
$$

(the solution with the negative sign being excluded for physical reasons). 
On the other hand you have

$$
c \mathbf{P}=E \mathbf{u} / c
$$

as a consequence of the definitions of $\mathbf{P}$ and $E$. Hence $m=0$ implies $u=c$ (and vice versa): particles of zero rest mass move with the limiting velocity $c$ (with respect to any inertial frame). - The inertial or relative mass is defined by

$$
m_{\mathrm{in}}={ }_{\mathrm{df}} m\left[1-u^{2} / c^{2}\right]^{-1 / 2}=E / c^{2} .
$$

Thus it is a function of either $u$ and $m$, or of $E$ alone. Since for $m=0 u=c$ the first expression is $0 / 0$, one has to use the second expression as the general definiens. Referents: all carriers of energy.

If you exclude from the referent class the electromagnetic field (or the photons) you violate the conservation laws. Let a battery (I) supply the energy to the radiator in the cavity (II). Then, as the book admits, $\Delta m_{\mathrm{I}}=-\Delta E / c^{2}$. Since the compound system $\mathrm{I}+\mathrm{II}$ is energetically and materially closed, $\Delta m_{\mathrm{II}}=-\Delta m_{\mathrm{I}}=\Delta E / c^{2}$. You can test this by putting battery and cavity on the same scale of your balance; according to SR the scales remain balanced during the process of energy transfer from battery to cavity, according to B.'s book the scale with the compound system goes up - another miracle in view of the fact that the compound system is closed.

Moral: Do not restrict the applicability (referent class) of a fundamental theory unless you have specific reasons; check for consistency of any such restriction.

\section{A SUBSTITUTE FOR QUANTUM MECHANICS (QM) ${ }^{7}$}

While the errors pointed out above can be corrected without affecting the substance of the book, the same cannot be said about the mistakes in the book's presentation of QM. In fact, what the book presents is not QM but a substitute suffering from the following major defects:

(1) inconsistencies, (2) (with the inconsistencies removed) zero or wrong physical content, depending on how the inconsistencies are removed. This is a disappointing and also surprising result, considering that the underlying conception has two sound components: (A) the objects to which QM refers are objects sui generis, neither waves nor corpuscles, properly called quantons, or systems of quantons; (B) QM is a probabilistic, not 
a statistical theory. The disease is in the third component: (C) probability $=$ probability of being $\left(\right.$ prob $_{1}$ ) [instead of: probability of becoming or transition $\left.\left(\mathrm{prob}_{2}\right)\right]$. There is a further malaise: the use of a representation (the Schroedinger representation) of Hilbert space (HS) whereby both the mathematics and the semantics of the theory are obscured: the distinction between state vectors and transition probability amplitudes is lost as the former are now represented by the latter. - So much by way of introduction. Now the details.

\subsection{Confusion About States}

14. ("All states are the same") " $Q M 5$ (c) If $U$ is a unitary operator..., then $U \Psi$ and $\Psi$ represent the same state..." (p. 246).

As the Schroedinger equation is equivalent to

$$
\Psi(t)=U(t) \Psi(0), U(t)=\exp \left(i H_{\mathrm{op}} t / \hbar\right)
$$

axiom $Q M 5$ (c) implies that a quanton does not change its state in the course of time. Further, since $U \Psi$ is an eigenstate of $U A_{\mathrm{op}} U^{-1}$ if $\Psi$ is an eigenstate of $A_{\text {op }}$, the axiom implies that the eigenstates of momentum and position are the same, $U$ in this case being the operator of a Fourier transformation. In fact, since unitary transformations are rotations in HS, the axiom implies that all states are the same.

Suggested explanation: Unitary covariance of fundamental equations has been misunderstood to mean semantic invariance of unitary transforms.

Advice: Scrap the axiom.

15. (Schroedinger equation) "Df.8. Any $\Psi \in \mathfrak{h}$ that satisfies (5.3) [Schroedinger equation] is called a state of the corresponding quanton" (p. 247). The Schroedinger equation defines a unitary operation (i.e., a mapping); it is not a functional equation that can, or cannot, be "satisfied".

Advise: Scrap it!

16. ("Pure" and "mixed" nonsense) "Df.9. Any $\Psi \in \mathfrak{h}$ ) satisfying (5.3) and such that $\Psi$ is not a linear combination of two other states is called a pure state." "Df.10. Any state that is not a pure state is called a mixture." (p. 247).

(1) HS being a linear vector space, any element $\Psi$ of HS can be written 
as a linear combination of other elements of HS. Hence Df.9 entails that there are no "pure states".

(2) In the context of one-system theory the terms 'pure state' and 'mixture' do not occur. If ensembles (virtual or real) are considered the correct terms are 'uniform ensemble' (all elements being in the same state) and 'nonuniform ensemble' or 'mixture' (not all elements in the same state). A probabilistic ensemble is a virtual non-uniform ensemble with given probabilities (theoretical weights) for the occurrence of the various states. A statistical ensemble is a real non-uniform ensemble with given relative frequencies for the various states. - If a uniform real ensemble is subjected to interaction with a stochastic state changer it turns into a statistical ensemble with relative frequencies to be compared with the theoretical weights given by the transition probabilities. If a single system is subjected to interaction with a stochastic state changer, its state changes into one of a set of possible states (just as a die does in die casting), the prob ${ }_{2}$ being given by the theory. - That is, basically, all there is to it. (The confusion in this matter is shared by many books).

Advise: Scrap Df.9 and Df.10.

\section{2. "Q-Densities" - The Ghosts That Never Appear}

17. " $Q M 3.2$ (f). If $Q_{\text {op }} \triangleq Q$, then the value of $\Psi^{*} Q_{\text {op }} \Psi$ at the point $(\sigma, \bar{\sigma}, x, t)$ represents the $Q$-density of the compound system $\sigma+\bar{\sigma}$ at $x, t$ "' (p. 246).

No objection to introducing names for mathematical expressions! Yet $Q M 3.2$ (f) is presented as a semantical axiom. Thus we must enquire what is meant by 'density'. We are told:

(1) The word 'density' is to be understood here in the sense of mathematical statistics [!] not of classical physics.

(2) $Q M$ 6a [which is QM 3.2(f) above with $Q=x=$ position] shows once again that, from a mathematical point of view, $Q M$ in the Schroedinger version is a field theory (p. 259).

Logically, you can have one or the other, physically you can have none of the two.

To see what the representatives of the "Q-densities" really mean or rather do not mean, one only has to integrate them, the result being

$$
\sum_{(k)}\left|\left(\Psi, \varphi_{k}\right)\right|^{2} q_{k}
$$




\section{CORRECTIONS TO BUNGE'S 'FOUNDATIONS OF PHYSICS'}

where $\varphi_{k}$ and $q_{k}$ are the eigenfunctions and eigenvalues of $Q_{\mathrm{op}}$. Now the factors of the $q_{k}$ are the well-known transition probabilities ${ }^{8}$

$$
\left|\left(\Psi, \varphi_{k}\right)\right|^{2}=\operatorname{prob}_{2}\left(\Psi \rightarrow \varphi_{k}\right) \text {. }
$$

They are invariant under unitary transformations and hence do not depend on the choice of the representation, while the "Q-densities" do not even exist in a non-Schroedinger representation. ${ }^{9}$

I have called 10 the "Q-densities" "ghosts that never appear except on paper'. I should add: they do not even appear on paper except in the Schroedinger representation.

Advise: Scrap the axioms quoted above. This makes place for introducing (2) as the correct semantical postulate.

Moral: Do not use a particular representation (coordinate system in HS) if everything fundamental is independent of it.

\section{SHORTCOMINGS}

18. Not, or not properly, discussed are the following fundamental questions:

(1) What is the number of independent physical dimensions in a given physical theory? Is this a syntactic, a semantic, or a syntacto-semantical problem?

(2) When are scale metrics conventions?

19. Intertheory relations between genetically connected theories should be discussed: this helps to clarify their characteristics.

20. The insistance on correct semantics should not blind one to the pragmatic aspects of a physical theory: physical theories are not only about some parts of nature, they are also for the human race to use.

\section{REFERENCES}

${ }^{1}$ M. Bunge, Foundations of Physics, Berlin, Heidelberg, New York, 1967.

2 M. Strauss, 'Grundlagen der Modernen Physik', in Mikrokosmos - Makrokosmos Bd. 2 (eds. H. Ley and R. Loether), Berlin 1967.

3 M. Strauss, 'Einstein's Theories and the Critics of Newton', in Synthese 18 (1968) 251-284; this paper may be consulted for a fuller discussion. 
${ }^{4}$ If the Lorentz force law (which does not belong to Maxwell's equations) is postulated the transformation properties of $(\mathbf{E}, \mathbf{B})$ can be deduced from those of force and velocity. ${ }^{5}$ M. Strauss, 1.c. ref. 3 , p. 268.

${ }^{6} \mathrm{M}$. Strauss, "Ist die Isotropie der Lichtausbreitung in einem Inertialsystem eine Konvention?', Monatsber. Dtsch. Akad. Wiss. Berlin 7 (1965) 626-627.

7 I do not know any text book on QM wholly correct in questions of logic (syntax and semantics) : : if I did $I$ would just say: look it up. For a concise statement of essentials, see M. Strauss, 1.c. ref. 2 and 'Restatement of QM' in the author's 'Intertheory Relations', to appear in the Proceedings of the 1968 Salzburg Colloquium in Philosophy of Science.

8 It follows that expression (1) is the mean value of the $q_{k}$ 's in a statistical ensemble (considered under [16]) in which the relative frequencies of states $k$ are equal to the theoretical weights. Thus, it is not "the expectation value of $\mathrm{Q}$ in state $\Psi$ " nor "the mean value of $\mathrm{Q}$ in state $\Psi$ ": these expressions are senseless, though they can be found in most books on QM, including B.'s (p. 255).

9 Incidentally, it is prob (transition probabilities) to which Popper's propensity interpretation applies: the probabilities in games of chance (such as die casting) considered by Popper are, basically, transition probabilities; in die casting the stochastic state changer is gravity-cum-table top; there is only one "quantity" being "measured", its "eigenvalues" being the figures ' 1 ' to ' 6 '.

10 M. Strauss, 1.c. ref. 7 . 